\title{
AN IMAGE PROCESSING APPROACH TO COARSE AGGREGATE FEATURE EXTRACTION IN HARDENED CONCRETE
}

\author{
Arsalaan Khan ${ }^{1 *}$, Muhammad Hashim ${ }^{2}$, Murad Khan ${ }^{3}$ Muhammad Atif Shamim ${ }^{4}$ \\ *1,2,3,4 Department of civil engineering, university of engineering \& technology, peshawar, pakistan \\ Engr.arsalaan@gmail.com²,mhashimkhan11@gmail.com², muradmkd99@gmail.com³,tifbarki61@gmail.com
}

*Corresponding Author: -

Email: Engr.arsalaan@gmail.com

\section{Samples}

from 1 inch down aggregate, 10 cylindrical samples were prepared in the laboratory according to ASTM gradation. This paper presents an automated digital image processing technique for capturing the microstructure of cement concrete. Cement concrete compound material of these three phases Aggregate, sand mastic and air void. Aggregate shape, gradation and orientation also play an important role. In this paper tried to detect and separate these three phases and measure the value of them by image processing technique.

\section{Methodology}

consists of Concrete Mix Design, Sample Preparation, Compressive Strength Test, Sample Cutting, Image Analysis, Extracting the Data Required, Data Analysis, Comparing the Results with ASTM Gradation Curve. Concrete mix design is the process of choosing suitable ingredient of concrete and determines their relative quantities with the object of producing as economically as possible concrete of certain minimum properties, notable workability, strength and durability. DC is a destructive technique, due to the need for cut sample surfaces and fails to capture the air void distribution but it has the advantage of capturing greater aggregate detail on an individual plane and being relatively inexpensive. As we are doing our project with destructive technique, therefore we cut the samples at different level. After the samples are prepared, each specimen was transversely sawed at the $1 / 2$ inch from bottom and top through core cutter to get the cross section of the sample. Core cutter is operated through electric power. In this way, we get cross section for each specimen at different level i-e top, $1 / 4$ (a) from top, 2/4 from top (b), 3/4 from top(c), 4/4 from the top (d) \& at bottom. After cutting of all the samples at different level of sample, Images are then acquired with digital camera (Xperia 8 megapixel) so that the different constituents of concrete should be clearly visible. Image analysis will be performed on these images with "Image $\mathrm{J}$ " the image analysis software. Analysis will be done in unit of inches. Image processing is a method to convert an image into digital form and perform some operation on it, in order to get an enhanced image or to extract some useful information from it. It is a process of meaningful information from images such as finding shapes, counting objects, identifying colors or measuring object properties. Extracting the data from images, the aggregate are labeled, and the data is exported to spreadsheet. After that finding the area and perimeter from the analysis data and make the graphs for the cross check of ASTM gradation curves. The digitally filtered images were utilized to calculate a gradation for the aggregate and compare with the real gradation of aggregate. The gradation of aggregate was determined by percentage retained in different sieve sizes according to volume. 